\title{
Influence of spin transfer and contact resistance on measurement of the spin Hall effect
}

\author{
Liangbin Hu, Ju Gao, and Shun-Qing Shen \\ Department of Physics, The University of Hong Kong, Pokfulam Road, Hong Kong, People's Republic of China
}

(Received 25 March 2003; revised manuscript received 26 June 2003; published 5 September 2003)

\begin{abstract}
When a longitudinal spin-unpolarized charge current flows in a thin slab of nonmagnetic metal with strong spin-orbit coupling of conduction electrons, a transverse spin Hall voltage can be generated between both edges of the sample, and the spin Hall voltage can be detected by the measurement of an ordinary Hall voltage produced in a transverse metal strip which connects both edges of the sample. In this paper, we discuss the influences of spin transfer and contact resistances between the longitudinal sample and the transverse metal strip on the measurement of the spin Hall voltage. We show that, due to the spin transfer between the longitudinal sample and the transverse metal strip, the magnitude of the spin Hall voltage produced in the longitudinal sample and the magnitude of the ordinary Hall voltage produced in the transverse metal strip and the relation between them will all strongly depend on the contact resistances, thus in order to detect the spin Hall voltage correctly through the measurement of the ordinary Hall voltage, the influences of spin transfer and contact resistance need to be taken into account.
\end{abstract}

DOI: 10.1103/PhysRevB.68.115302

PACS number(s): 73.61.At, 72.15.Gd, 73.50.Jt

\section{INTRODUCTION}

It was proposed by Hirsch that when a longitudinal spinunpolarized charge current flows in a thin slab of nonmagnetic metal with strong spin-orbit coupling of moving conduction electrons, a transverse spin imbalance may be caused in the slab, i.e., at both sides of the slab nonequilibrium spin accumulation may occur. ${ }^{1}$ This effect was named the spin Hall effect in Ref. 1, and was discussed in some more detail in Ref. 2. The spin Hall effect is very different from the spin accumulation effect found in magnetic multilayers when a charge current flows in a direction perpendicular to the plane of the layers, which is caused by the discontinuity of material properties at the interfaces of alternating layers. ${ }^{3-6}$ From theoretical viewpoints, the spin Hall effect arises from the spinorbit-coupling induced left-right asymmetric scattering of moving conduction electrons. The spin-orbit-coupling induced left-right asymmetric scattering was known as skew scattering in the literature, and was believed to be the origin of the anomalous Hall coefficients experimentally found in ferromagnetic metals. ${ }^{7,8}$ In the spin Hall effect, as illustrated in Fig. 1(a), when a longitudinal spin-unpolarized charge current density $j_{x}$ flows in a thin slab of nonmagnetic metal, due to the spin-orbit-coupling induced skew scattering, spin-up electrons will have a larger probability to be scattered to the right and spin-down electrons will have a larger probability to be scattered to the left. This left-right asymmetric scattering will cause a transverse spin imbalance in the slab, and results in a nonequilibrium spin accumulation at both sides of the slab, but no charge imbalance will occur in the slab. This is very different from the ordinary Hall effect. In the ordinary Hall effect, the Lorentz forces felt by moving conduction electrons in external magnetic fields will cause a transverse charge imbalance in a sample, and results in charge accumulation at both sides of a sample, but no spin imbalance will occur. In the ordinary Hall effect, the Fermi levels for spin-up and spin-down electrons are the same, but due to the occurrence of transverse charge imbalance, the Fermi levels for electrons at both edges of the sample are different, and the difference in the Fermi levels at both edges of the sample is defined as the Hall voltage, which can be measured by a voltmeter. In the spin Hall effect, due to the occurrence of transverse spin imbalance at both sides of the sample, the Fermi levels for each spin at both edges of the

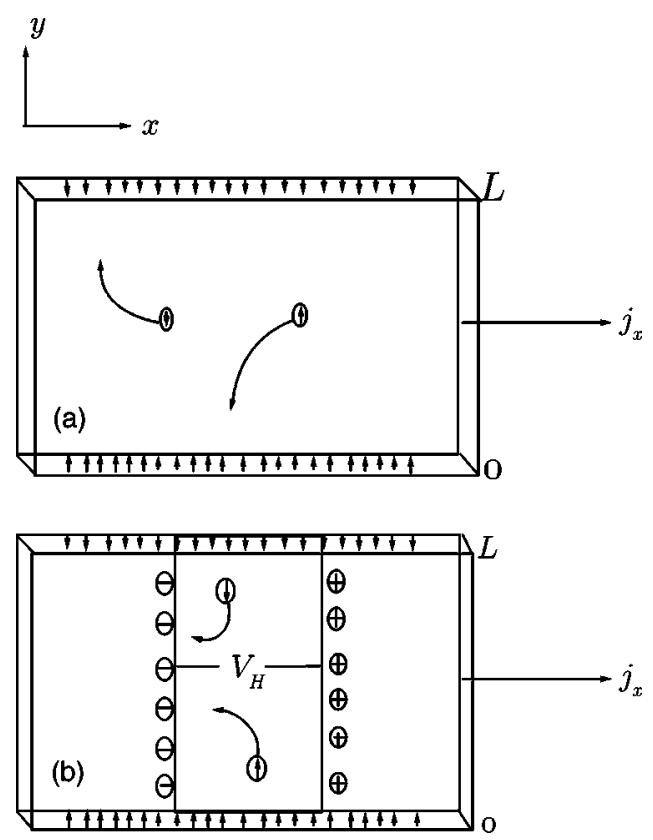

FIG. 1. (a) When a longitudinal spin-unpolarized charge current flows in a thin slab of nonmagnetic metal with spin-orbit coupling of conduction electrons, due to the spin-orbit-coupling induced skew scattering, spin-up electrons will have a larger probability to be scattered to the right and spin-down electrons a larger probability to be scattered to the left, leading to a transverse spin imbalance in the slab. (b) If both edges of the slab are connected by a transverse metal strip, a longitudinal spin current will flow in the strip. If the skew scattering mechanism also operates in the strip, the longitudinal spin current that flows in the strip will cause a transverse charge imbalance in the strip, and hence an ordinary Hall voltage will be produced between both edges of the strip. 
sample are different, and, similar to the ordinary Hall voltage, the difference in the Fermi levels for each spin at both edges of the sample can be defined as the spin Hall voltage; however, it cannot be measured directly by ordinary voltmeters. In order to detect the spin Hall voltage by ordinary voltmeters, it was proposed in Ref. 1 that one can connect both edges of the sample by a transverse metal strip and let the transverse metal strip contact the sample only at both edges. Due to the existence of the differences in the Fermi levels for each spin at both edges of the sample, a longitudinal spin current will flow in the transverse metal strip. Providing that the same skew scattering mechanism also operates in the transverse metal strip, the longitudinal spin current in the strip will cause a transverse charge imbalance in the strip; thus an ordinary charge Hall voltage will be produced between both edges of the strip, which can be measured by ordinary voltmeters. [It should be pointed out that the meaning of the terminology "Hall voltage" used here is a little different from the usual one. In the usual Hall effect, the Hall voltage is caused by the Lorentz forces felt by moving conduction electrons in a perpendicular magnetic field, and the voltage occurs in the direction perpendicular to the applied charge current. The Hall voltage discussed here is induced by the spin-orbit coupling of moving conduction electrons, and the voltage occurs in the same direction as the applied charge current $j_{x}$, as shown in Fig. 1(b).] According to the picture described above, in principle one can detect the spin Hall voltage $V_{S H}$ between both edges of the longitudinal sample through the measurement of the ordinary Hall voltage $V_{H}$ between both edges of the transverse metal strip, providing that the relation between $V_{S H}$ and $V_{H}$ is known. This relation was derived theoretically in Ref. 1. It is anticipated that such an experiment and the findings resulting from it could find some practical applications in the emerging field of spintronics. ${ }^{1,9}$

In the present paper, we discuss the influences of spin transfer and contact resistances between the longitudinal sample and the transverse metal strip on the measurement of the spin Hall effect. Such influences were not considered in Ref. 1, but in real experiments there will inevitably exist spin transfer and contact resistances between the longitudinal sample and the transverse metal strip. In this paper we will show that the spin transfer and contact resistances between the longitudinal sample and the transverse metal strip will have significant influences on the measurement of the spin Hall effect. We will show that, due to the spin transfer between the longitudinal sample and the transverse metal strip, both the magnitudes of the spin Hall voltage $V_{S H}$ produced in the longitudinal sample and the ordinary Hall voltage $V_{H}$ produced in the transverse metal strip and the relation between them will all strongly depend on the contact resistances; thus, in order to detect the spin Hall voltage $V_{S H}$ through the measurement of the ordinary Hall voltage $V_{H}$ correctly, the influences of spin transfer and contact resistances need to be taken into account.

The paper is organized as follows: In Sec. II, we will derive some general expressions for the spin Hall voltage $V_{S H}$ produced in the longitudinal sample and the ordinary Hall voltage $V_{H}$ produced in the transverse metal strip and the relation between them by taking into account the influences of spin transfer and contact resistances. In Sec. III, the influences of spin transfer and contact resistances will be discussed in detail in two specialy interesting cases.

\section{DERIVATION OF THE SPIN HALL VOLTAGE $V_{S H}$ AND THE ORDINARY HALL VOLTAGE $V_{H}$ IN THE PRESENCE OF SPIN TRANSFER AND CONTACT RESISTANCES}

The system is described schematically in Fig. 1(b). In the following we will denote the width of the longitudinal sample as $L$, and will assume that the two boundaries of the longitudinal sample are located at $y= \pm L / 2$. The width of the transverse metal strip will be denoted as $l$, and it will be assumed that $l$ is sufficiently small so that the voltage drop along the contacts can be neglected. The skew scattering mechanism is assumed to operate both in the longitudinal sample and in the transverse metal strip. After taking into account the skew scattering mechanism, the spin-dependent charge current densities in the longitudinal sample and in the transverse metal strip can be expressed as ${ }^{1,2}$

$$
\begin{gathered}
\vec{j}^{(\sigma)}(\vec{r})=C \vec{E}^{\sigma}(\vec{r})+C_{H} \vec{E}^{\sigma}(\vec{r}) \times \vec{\sigma}, \\
\vec{j}^{(\sigma)}(\vec{r})=C^{\prime} \vec{E}^{\prime \sigma}(\vec{r})+C_{H}^{\prime} \vec{E}^{\prime \sigma}(\vec{r}) \times \vec{\sigma},
\end{gathered}
$$

where $\vec{E}^{\sigma}$ and ${\overrightarrow{E^{\prime}}}^{\sigma}$ are the spin-dependent effective fields in the longitudinal sample and in the transverse metal strip, respectively; $C$ and $C^{\prime}$ are the ordinary conductivities of the longitudinal sample and the transverse metal strip, respectively; and $C_{H}$ and $C_{H}^{\prime}$ are the spin-orbit-coupling induced anomalous Hall conductivities of the longitudinal sample and the transverse strip, respectively. The spin-orbit-coupling induced anomalous Hall conductivities are usually much smaller than the ordinary conductivities of a sample, i.e., $C_{H} \ll C$ and $C_{H}^{\prime} \ll C^{\prime}$. Due to the occurrence of the transverse spin imbalance in the longitudinal sample and the occurrence of a transverse charge imbalance in the transverse metal strip, the spin-dependent effective fields felt by conduction electrons in the longitudinal sample and in the transverse metal strip will be given by

$$
\begin{gathered}
\vec{E}^{(\sigma)}=\frac{j_{x}}{C} \vec{e}_{x}-\frac{\partial \mu_{\sigma}}{\partial y} \vec{e}_{y}, \\
\vec{E}^{\prime(\sigma)}=-\frac{V_{H}}{l} \vec{e}_{x}-\frac{\partial \mu_{\sigma}^{\prime}}{\partial y} \vec{e}_{y},
\end{gathered}
$$

where $j_{x}$ is the applied longitudinal charge current density in the sample, $V_{H}$ is the ordinary Hall voltage produced between both edges of the transverse metal strip, and $\mu_{\sigma}$ and $\mu_{\sigma}^{\prime}$ are the position and spin dependent shifts of the Fermi levels in the longitudinal sample and in the transverse metal strip, respectively. Substituting Eq. (3) into Eq. (1), the transverse components of the spin-dependent charge current densities in the longitudinal sample can be expressed as 


$$
\begin{aligned}
& j_{y}^{(\uparrow)}=-C \frac{\partial \mu_{\uparrow}}{\partial y}-\frac{C_{H}}{C} j_{x}, \\
& j_{y}^{(\downarrow)}=-C \frac{\partial \mu_{\downarrow}}{\partial y}+\frac{C_{H}}{C} j_{x} .
\end{aligned}
$$

Similarly, substituting Eq. (4) into Eq. (2), the spindependent charge current densities generated in the transverse metal strip can be expressed as

$$
\begin{gathered}
j_{y}^{\prime(\sigma)}=-C^{\prime} \frac{\partial \mu_{\sigma}^{\prime}}{\partial y} \quad(\sigma=\uparrow, \downarrow), \\
j_{x}^{\prime(\uparrow)}=-C_{H}^{\prime} \frac{\partial \mu_{\uparrow}^{\prime}}{\partial y}-C^{\prime} \frac{V_{H}}{l}, \\
j_{x}^{\prime(\downarrow)}=C_{H}^{\prime} \frac{\partial \mu_{\downarrow}^{\prime}}{\partial y}-C^{\prime} \frac{V_{H}}{l} .
\end{gathered}
$$

In a steady state, the $x$ component of the total charge current density in the transverse metal strip should be zero, i.e., $j_{x}^{\prime(\uparrow)}+j_{x}^{\prime(\downarrow)}=0$. From this condition the ordinary Hall voltage $V_{H}$ can be expressed as

$$
V_{H}=\frac{l C_{H}^{\prime}}{2\left(C^{\prime}\right)^{2}}\left(j_{y}^{\prime(\uparrow)}-j_{y}^{\prime(\downarrow)}\right) \text {. }
$$

Since no charge imbalance occurs in the $y$ direction of the system, then both in the longitudinal sample and in the transverse metal trip the $y$ components of the total charge current densities should be zero ${ }^{10}$ :

$$
\begin{aligned}
& j_{y}^{(\uparrow)}+j_{y}^{(\downarrow)}=0, \\
& j_{y}^{\prime(\uparrow)}+j_{y}{ }^{(\downarrow)}=0 .
\end{aligned}
$$

The boundary conditions at the contacts between the longitudinal sample and the transverse metal strip can be written down from the generalized Ohm's law by taking into account the contact resistances (or equivalently, the contact conductivities). ${ }^{4,11}$ The contact resistances (conductivities) are caused by the interfacial scattering of electrons at the contacts between the longitudinal sample and the transverse metal strip. In the presence of both spin-conserving and spinflip interfacial scattering, two types of contact conductivities need to be considered, i.e., the "spin-conserving" contact conductivities and the "spin-flip" contact conductivities. ${ }^{11}$ In the following we will denote the spin-conserving contact conductivity between the longitudinal sample and the transverse metal strip as $\Sigma_{c}$ and the spin-flip contact conductivities as $\Sigma_{c}^{\prime}$, which describe, respectively, the spin-conserving and spin-flip transfers of electrons between the longitudinal sample and the transverse metal strip. (Since it has been assumed that the longitudinal sample and the transverse metal strip are both nonmagnetic, one can assume that $\Sigma_{c}$ and $\Sigma_{c}^{\prime}$ are spin independent). According to the picture described in Fig. 1(b), at the contact at $y=-L / 2$ the spin-up charge current will be transferred from the longitudinal sample into the transverse metal strip, and the spin-down charge current will be transferred from the transverse metal strip into the longitudinal sample. Similarly, at the contact at $y=L / 2$, the spin-up charge current will be transferred from the transverse metal strip into the longitudinal sample and the spin-down charge current will be transferred from the longitudinal sample into the transverse metal strip. Considering these facts and by use of the generalized Ohm's law, the boundary conditions can be written down as follows:

$$
\begin{aligned}
l j_{y=-L / 2}^{(\downarrow)}= & \Sigma_{c}\left[\mu_{\downarrow}^{\prime}\left(-\frac{L}{2}\right)-\mu_{\downarrow}\left(-\frac{L}{2}\right)\right] \\
& +\Sigma_{c}^{\prime}\left[\mu_{\uparrow}^{\prime}\left(-\frac{L}{2}\right)-\mu_{\downarrow}\left(-\frac{L}{2}\right)\right], \\
l j_{y=-L / 2}^{\prime(\uparrow)}= & \Sigma_{c}\left[\mu_{\uparrow}\left(-\frac{L}{2}\right)-\mu_{\uparrow}^{\prime}\left(-\frac{L}{2}\right)\right] \\
& +\Sigma_{c}^{\prime}\left[\mu_{\downarrow}\left(-\frac{L}{2}\right)-\mu_{\uparrow}^{\prime}\left(-\frac{L}{2}\right)\right],
\end{aligned}
$$

$$
-l j_{y=L / 2}^{(\uparrow)}=\Sigma_{c}\left[\mu_{\uparrow}^{\prime}\left(\frac{L}{2}\right)-\mu_{\uparrow}\left(\frac{L}{2}\right)\right]+\Sigma_{c}^{\prime}\left[\mu_{\downarrow}^{\prime}\left(\frac{L}{2}\right)-\mu_{\uparrow}\left(\frac{L}{2}\right)\right],
$$

$$
-l j_{y=L / 2}^{\prime(\downarrow)}=\Sigma_{c}\left[\mu_{\downarrow}\left(\frac{L}{2}\right)-\mu_{\downarrow}^{\prime}\left(\frac{L}{2}\right)\right]+\Sigma_{c}^{\prime}\left[\mu_{\uparrow}\left(\frac{L}{2}\right)-\mu_{\downarrow}^{\prime}\left(\frac{L}{2}\right)\right] .
$$

There is a negative sign on the left-hand side of Eqs. (15) and (16) since we have defined that the values of $j_{y}^{(\sigma)}$ (or $'(\sigma)$

$j_{y}$ ) will be negative if the current flows in the negative $y$ direction. If no spin-flip interfacial scattering exist at the contacts (i.e., the "spin-flip" contact conductivity $\Sigma_{c}^{\prime}=0$ ), then from Eqs. (11) and (12) and (13)-(16) one can show that the spin current will be conserved when it is transferred from the longitudinal sample into the transverse metal strip (or vice versa), i.e., $j_{y}^{(\uparrow)}-j_{y}^{(\downarrow)}=j_{y}^{\prime(\uparrow)}-j_{y}^{\prime(\downarrow)}$ at $y= \pm L / 2$.

From Eqs. (5)-(9), the spin-dependent charge current densities (both in the longitudinal sample and in the transverse metal strip) can be obtained if $\partial \mu_{\sigma} / \partial y$ and $\partial \mu_{\sigma}^{\prime} / \partial y$ are known. If the width $L$ of the sample is much smaller than the spin-diffusion lengths, $\partial \mu_{\sigma} / \partial y$ and $\partial \mu_{\sigma}^{\prime} / \partial y$ can be approximated as $\partial \mu_{\uparrow} / \partial y=-V_{S H} / L, \quad \partial \mu_{\downarrow} / \partial y=V_{S H} / L, \quad \partial \mu_{\uparrow}^{\prime} / \partial y$ $=-V_{S H}^{\prime} / L$, and $\partial \mu_{\downarrow}^{\prime} / \partial y=V_{S H}^{\prime} / L$, in which $V_{S H}$ is the spin Hall voltage between both edges of the longitudinal sample and $V_{S H}^{\prime}$ is the spin Hall voltage between both ends of the transverse metal strip. This is the case discussed in Ref. 1. To make our discussion more general, we will not confine our discussion to the case in which the width $L$ of the sample is much smaller than the spin-diffusion lengths. In general cases, $\mu_{\sigma}$ and $\mu_{\sigma}^{\prime}$ will satisfy the following spin-diffusion equations $^{3,4}$ : 


$$
\begin{aligned}
& \nabla^{2}\left[\mu_{\uparrow}-\mu_{\downarrow}\right]=\frac{\mu_{\uparrow}-\mu_{\downarrow}}{D^{2}}, \\
& \nabla^{2}\left[\mu_{\uparrow}^{\prime}-\mu_{\downarrow}^{\prime}\right]=\frac{\mu_{\uparrow}^{\prime}-\mu_{\downarrow}^{\prime}}{D^{\prime 2}},
\end{aligned}
$$

where $D$ and $D^{\prime}$ are the spin-diffusion length in the longitudinal sample and in the transverse metal strip, respectively. For simplicity, in the following we will assume that $D$ $=D^{\prime}$. From Eqs. (17) and (18) and with the help of Eqs. (11) and (12), one can show that $\mu_{\uparrow}(y), \mu_{\downarrow}(y), \mu_{\uparrow}^{\prime}(y)$, and $\mu_{\downarrow}^{\prime}(y)$ can be expressed as

$$
\begin{gathered}
\mu_{\uparrow}(y)=-\mu_{\downarrow}(y)=A e^{y / D}+B e^{-y / D}, \\
\mu_{\uparrow}^{\prime}(y)=-\mu_{\downarrow}^{\prime}(y)=A^{\prime} e^{y / D}+B^{\prime} e^{-y / D},
\end{gathered}
$$

where $A, B, A^{\prime}$, and $B^{\prime}$ are constant coefficients. Substituting Eqs. (19) and (20) into Eqs. (5)-(9), the spin-dependent charge current densities $\vec{j}^{(\sigma)}$ and $\vec{j}^{(\sigma)}$ can be expressed as functions of these constant coefficients, then by use of conditions (13)-(16), these constant coefficients can be determined, and the results can be expressed as

$$
\begin{aligned}
& A=-B=-\frac{\rho_{H} j_{x} l D\left[\rho_{c} \rho_{c}^{\prime} l+D \rho^{\prime}\left(\rho_{c}+\rho_{c}^{\prime}\right) \tanh \left(\frac{L}{2 D}\right)\right]}{\rho_{c} \rho_{c}^{\prime} l^{2} \cosh \left(\frac{L}{2 D}\right)+l D\left(\rho_{c}+\rho_{c}^{\prime}\right)\left(\rho+\rho^{\prime}\right) \sinh \left(\frac{L}{2 D}\right)+4 D^{2} \rho \rho^{\prime} \tanh \left(\frac{L}{2 D}\right) \sinh \left(\frac{L}{2 D}\right)}, \\
& A^{\prime}=-B^{\prime}=-\frac{\rho^{\prime} \rho_{H} j_{x} l D^{2}\left(\rho_{c}^{\prime}-\rho_{c}\right) \tanh \left(\frac{L}{2 D}\right)}{\rho_{c} \rho_{c}^{\prime} l^{2} \cosh \left(\frac{L}{2 D}\right)+l D\left(\rho_{c}+\rho_{c}^{\prime}\right)\left(\rho+\rho^{\prime}\right) \sinh \left(\frac{L}{2 D}\right)+4 D^{2} \rho \rho^{\prime} \tanh \left(\frac{L}{2 D}\right) \sinh \left(\frac{L}{2 D}\right)},
\end{aligned}
$$

where $\rho_{c} \equiv 1 / \Sigma_{c}$ and $\rho_{c}^{\prime} \equiv 1 / \Sigma_{c}^{\prime}$ are the spin-conserving and spin-flip contact resistivities, respectively; $\rho \equiv 1 / C$ and $\rho^{\prime} \equiv 1 / C^{\prime}$ are the ordinary resistivities of the longitudinal sample and the transverse metal strip, respectively; and $\rho_{H} \equiv C_{H} / C^{2}$ and $\rho_{H}^{\prime}$ $\equiv C_{H}^{\prime} / C^{\prime 2}$ are the anomalous Hall resistivities of the longitudinal sample and the transverse strip, respectively. ${ }^{12}$ After the coefficients $A, B, A^{\prime}$, and $B^{\prime}$ are determined, the spin Hall voltage $V_{S H}$ between both edges of the longitudinal sample can be obtained directly from Eq. (19), and the result can be expressed as

$$
V_{S H}=\frac{2 \rho_{H} j_{x} l D\left[\rho_{c} \rho_{c}^{\prime} l+D \rho^{\prime}\left(\rho_{c}+\rho_{c}^{\prime}\right) \tanh \left(\frac{L}{2 D}\right)\right] \sinh \left(\frac{L}{2 D}\right)}{\rho_{c} \rho_{c}^{\prime} l^{2} \cosh \left(\frac{L}{2 D}\right)+l D\left(\rho_{c}+\rho_{c}^{\prime}\right)\left(\rho+\rho^{\prime}\right) \sinh \left(\frac{L}{2 D}\right)+4 D^{2} \rho \rho^{\prime} \tanh \left(\frac{L}{2 D}\right) \sinh \left(\frac{L}{2 D}\right)} .
$$

The ordinary Hall voltage $V_{H}$ between both edges of the transverse metal strip can be obtained from Eq. (10), and the result can be expressed as

$$
V_{H}=\frac{\rho_{H} \rho_{H}^{\prime} j_{x} l^{2} D\left(\rho_{c}^{\prime}-\rho_{c}\right) \tanh (L / 2 D) \cosh (y / D)}{\rho_{c} \rho_{c}^{\prime} l^{2} \cosh \left(\frac{L}{2 D}\right)+D l\left(\rho_{c}+\rho_{c}^{\prime}\right)\left(\rho+\rho^{\prime}\right) \sinh \left(\frac{L}{2 D}\right)+4 D^{2} \rho \rho^{\prime} \tanh \left(\frac{L}{2 D}\right) \sinh \left(\frac{L}{2 D}\right)} .
$$

From Eqs. (23) and (24), one can see that the spin Hall voltage $V_{S H}$ between both edges of the longitudinal and the ordinary Hall voltage $V_{H}$ between both edges of the transverse metal strip satisfy the following relation:

$$
V_{S H}=\frac{2\left[\rho_{c} \rho_{c}^{\prime} l+D \rho^{\prime}\left(\rho_{c}+\rho_{c}^{\prime}\right) \tanh \left(\frac{L}{2 D}\right)\right] \cosh \left(\frac{L}{2 D}\right)}{\rho_{H}^{\prime} l\left(\rho_{c}^{\prime}-\rho_{c}\right) \cosh (y / D)} V_{H} .
$$

Using this relation, the magnitude of the spin Hall voltage $V_{S H}$ can be deduced from the magnitude of the ordinary Hall voltage $V_{H}$, which can be measured by ordinary voltmeters.

\section{RESULTS AND DISCUSSIONS}

From Eqs. (23)-(25), one can see that after taking into account the influences of spin transfer and contact resistances between the longitudinal sample and the transverse metal strip, both the magnitudes of $V_{S H}$ and $V_{H}$ and the relation 
between them will all strongly depend on the contact resistances, so in general spin transfer and contact resistances may have some significant influences on the measurement of the spin Hall effect. Two limiting cases will be especially interesting. The first case is that the width $L$ of the sample is much smaller than the spin-diffusion length $(L \ll D)$. This is the case considered in Ref. 1 . The second case is that the width $L$ of the sample is much larger than the spin-diffusion length $(L \gg D)$, which is not considered in Ref. 1. In the first case $(L \ll D)$, the ordinary Hall voltage between both edges of the transverse metal strip and the spin Hall voltage between both edges of the longitudinal sample and the relation between them will be given by

$$
\begin{aligned}
V_{H} & =\frac{\rho_{H} \rho_{H}^{\prime} \rho_{c} j_{x} l^{2} L(1-\gamma)}{2 \rho_{c}^{2} l^{2}+\rho_{c} l L(1+\gamma)\left(\rho+\rho^{\prime}\right)+2 \gamma \rho \rho^{\prime} L^{2}}, \\
V_{S H} & =\frac{\rho_{H} j_{x} l L\left[2 \rho_{c}^{2} l+\rho^{\prime} \rho_{c} L(1+\gamma)\right]}{2 \rho_{c}^{2} l^{2}+\rho_{c} l L(1+\gamma)\left(\rho+\rho^{\prime}\right)+2 \gamma \rho \rho^{\prime} L^{2}} \\
& =\frac{2 \rho_{c}^{2} l+\rho^{\prime} \rho_{c} L(1+\gamma)}{\rho_{H}^{\prime} \rho_{c} l(1-\gamma)} V_{H},
\end{aligned}
$$

where $\gamma\left(\equiv \rho_{c} / \rho_{c}^{\prime}=\Sigma_{c}^{\prime} / \Sigma_{c}\right)$ is the ratio of the spinconserving contact resistivity to the spin-flip contact resistivity (or equivalently, the ratio of the spin-flip contact conductivity to the spin-conserving contact conductivity). This ratio characterizes the relative strength of spin-flip interfacial scattering at the contacts. By neglecting the influences of spin transfer and contact resistances, the following results were be obtained in Ref. 1:

$$
\begin{gathered}
V_{H}=\frac{\rho_{H} \rho_{H}^{\prime}}{\rho^{\prime}} j_{x} l, \\
V_{S H}=\rho_{H} j_{x} L=\frac{\rho^{\prime} L}{\rho_{H}^{\prime} l} V_{H} .
\end{gathered}
$$

It can be seen that there are some significant differences between the corresponding results described in Eqs. (26) and (27) and (28) and (29). First, from Eqs. (26) and (27), one can see that after taking into account the influences of spin transfer and contact resistances, the dependences of $V_{S H}$ and $V_{H}$ on the ordinary resistivity $\rho$ and $\rho^{\prime}$ and the widths $L$ and $l$ of the longitudinal sample and the transverse metal strip are very different from those described in Eqs. (28) and (29). According to Eq. (28), the ordinary Hall voltage $V_{H}$ between both edges of the transverse metal strip should be independent of the ordinary resistivity $\rho$ and the width $L$ of the longitudinal sample, however, according to Eq. (26), $V_{H}$ will strongly depend on $\rho$ and $L$. Similarly, according to Eq. (29), $V_{S H}$ should be independent of the ordinary resistivity $\rho^{\prime}$ and the width $l$ of the transverse metal strip, but, according to Eq. (27), $V_{S H}$ will strongly depend on $\rho^{\prime}$ and $l$. It should be pointed out that Eqs. (26) and (27) are not equivalent to Eqs. (28) and (29) even in the ideal case that no any interfacial scattering exists at the contacts between the longitudinal sample and the transverse metal strip. In such an ideal case, Eqs. (26) and (27) will become $V_{H}=\rho_{H} \rho_{H}^{\prime} j_{x} l /\left(\rho+\rho^{\prime}\right)$ and $V_{S H}=\rho^{\prime} \rho_{H} j_{x} L /\left(\rho+\rho^{\prime}\right)$, which are also significantly different from Eqs. (28) and (29). This difference arises from the fact that the influences of the boundary conditions were neglected completely in Ref. 1 . The second and more important difference between Eqs. (26) and (27) and (28) and (29) is that, after taking into account the influences of spin transfer and contact resistances, both the magnitudes of $V_{S H}$ and $V_{H}$ and the relation between them will all strongly depend on the contact resistances. To obtain an estimate of the magnitudes of $V_{S H}$ and $V_{H}$ and their dependences on the contact resistances, let us consider some actual experimental parameters. For simplicity, we assume that the longitudinal sample and the transverse metal strip are made of the same material, for example, Al. At low temperature the spin diffusion length in $\mathrm{Al}$ is of the order of $450 \mu \mathrm{m}$, and the resistivity $\rho$ is of the order of $2.7 \times 10^{-3} \mu \Omega \mathrm{cm}$. As in Ref. 1, we assume that the spin-orbit-coupling induced anomalous Hall resistivity can be given by $\rho_{H}=2 \pi R_{S} n \mu_{B}$, in which $R_{S}$ is the anomalous Hall coefficient and $n$ is the density of electrons and $\mu_{B}$ is the Bohr magneton. The anomalous Hall coefficient $R_{S}$ is simply assumed to be the same as the ordinary Hall coefficient of Al, $R_{0}=3.45 \times 10^{-11} \mathrm{~m}^{3} / \mathrm{C}$. The width $L$ of the longitudinal sample will be taken to be $100 \mu \mathrm{m}$ (much smaller than the spin-diffusion length in $\mathrm{Al}$ ), the width $l$ of the transverse metal strip will be taken to be $50 \mu \mathrm{m}$, and the longitudinal charge current density $j_{x}$ will be taken to be 6 $\times 10^{6} \mathrm{~A} / \mathrm{m}^{2}$. For clarity, we first assume that no spin-flip interfacial scattering exists at the contacts between the longitudinal sample and the transverse metal strip (i.e., the spinflip contact conductivity $\Sigma_{c}^{\prime}$ is zero). In Figs. 2(a) and 2(b) we have plotted the changes of the magnitudes of $V_{S H}$ and $V_{H}$ with the variation of the spin-conserving contact resistivity $\rho_{c}$ (in units of the resistivity of $\mathrm{Al}$ ) using the experimental parameters listed above. From Figs. 2(a) and 2(b), one can see that in the ideal case of $\rho_{c}=0$, the ordinary Hall voltage $V_{H}$ between both edges of the transverse metal strip will be maximum but the spin Hall voltage $V_{S H}$ between both edges of the longitudinal sample will be minimum, $V_{H}$ will $d e$ crease with the increase of $\rho_{c}$, and $V_{S H}$ will increase with the increase of $\rho_{c}$. If $\rho_{c}$ is very large, the spin Hall voltage $V_{S H}$ between both edges of the longitudinal sample will reach its maximum value, but the ordinary Hall voltage $V_{H}$ between both edges of the transverse metal strip will become actually zero. Such features can also be seen from Eqs. (26) and (27), from which one can show that $V_{S H} \rightarrow \rho_{H} j_{x} L$ and $V_{H} \rightarrow 0$ in the limit of $\rho_{c} \rightarrow \infty$. The above results show that spin transfer and contact resistances may have some significant influences on the measurement of the spin Hall effect. First, the above results show that in general cases one cannot detect directly the magnitude of the spin Hall voltage $V_{S H}$ from the measured ordinary Hall voltage $V_{H}$ if the contact resistances are not known. In order to detect the magnitude of the spin Hall voltage $V_{S H}$ from the measured ordinary Hall voltage $V_{H}$ correctly, one must first measure the contact resistances (or conductivities). In principle, the contact resis- 

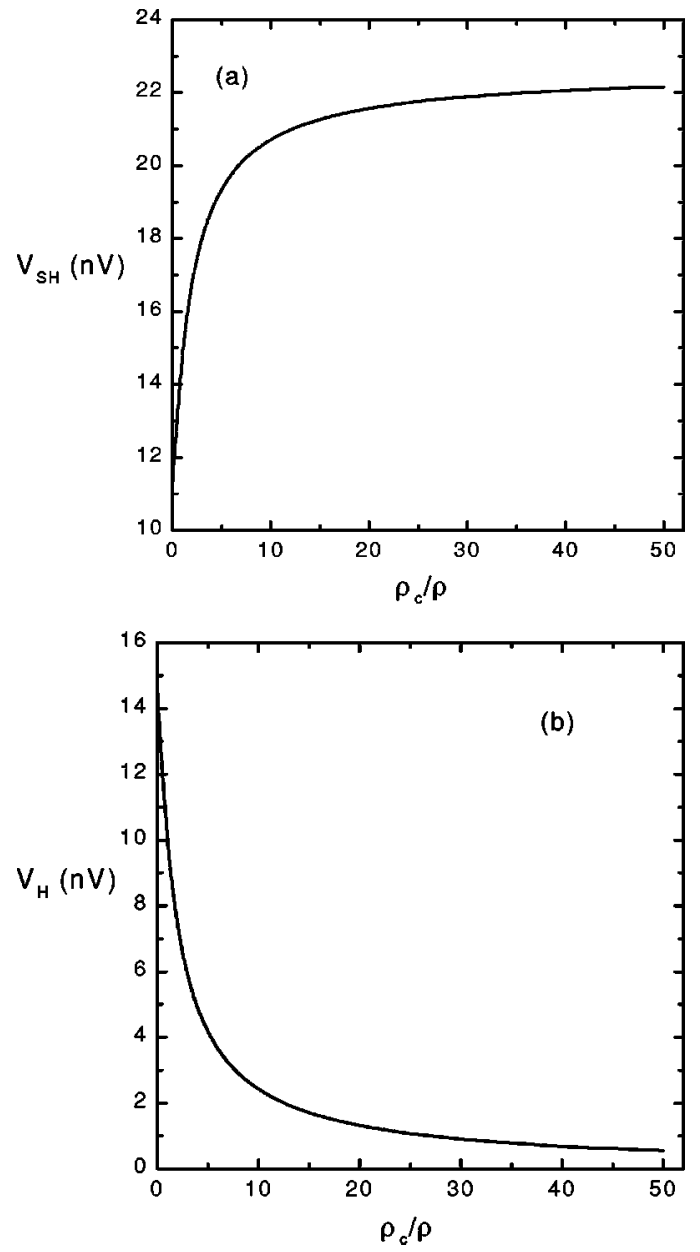

FIG. 2. Illustration of the changes of (a) the spin Hall voltage $V_{S H}$ and (b) the ordinary Hall voltage $V_{H}$ with the variation of the contact resistivity $\rho_{c}$ (in units of $\rho$, the resistivity of Al). The parameters used have been given in the text.

tances can be determined from the dependence of the ordinary Hall voltage $V_{H}$ on the applied longitudinal charge current density $j_{x}$ with the help of Eq. (24). After the contact resistances are determined, then one can determine the magnitude of the spin Hall voltage $V_{S H}$ from the measured ordinary Hall voltage $V_{H}$ by the use of Eq. (25). Second, the above results show that if the contact resistances are very large, the ordinary Hall voltage $V_{H}$ may be very small and not large enough to actually be measurable. For instance, for the example illustrated in Figs. 2(a) and 2(b), the ordinary Hall voltage $V_{H}$ is $14.5 \mathrm{~V}$ in the ideal case of $\rho_{c}=0$. This voltage is large enough to actually be measurable. If $\rho_{c}$ is nonzero but not very large, $V_{H}$ will still be large enough to be measurable. But if $\rho_{c}$ is as large as $0.1 \mu \Omega \mathrm{cm}, V_{H}$ will become smaller than $0.1 \mathrm{nV}$. Such a small voltage is not easily measurable. In such cases, it may be very difficult to detect the spin Hall voltage $V_{S H}$ through the measurement of the ordinary Hall voltage $V_{H}$. Finally, let us consider the influences of spin-flip interfacial scattering. In Figs. 3(a) and 3(b) we have plotted the changes of the spin Hall voltage $V_{S H}$ and the ordinary Hall voltage $V_{H}$ with the variation of the ratio $\gamma$ (i.e., the ratio of the spin-flip contact conductivity
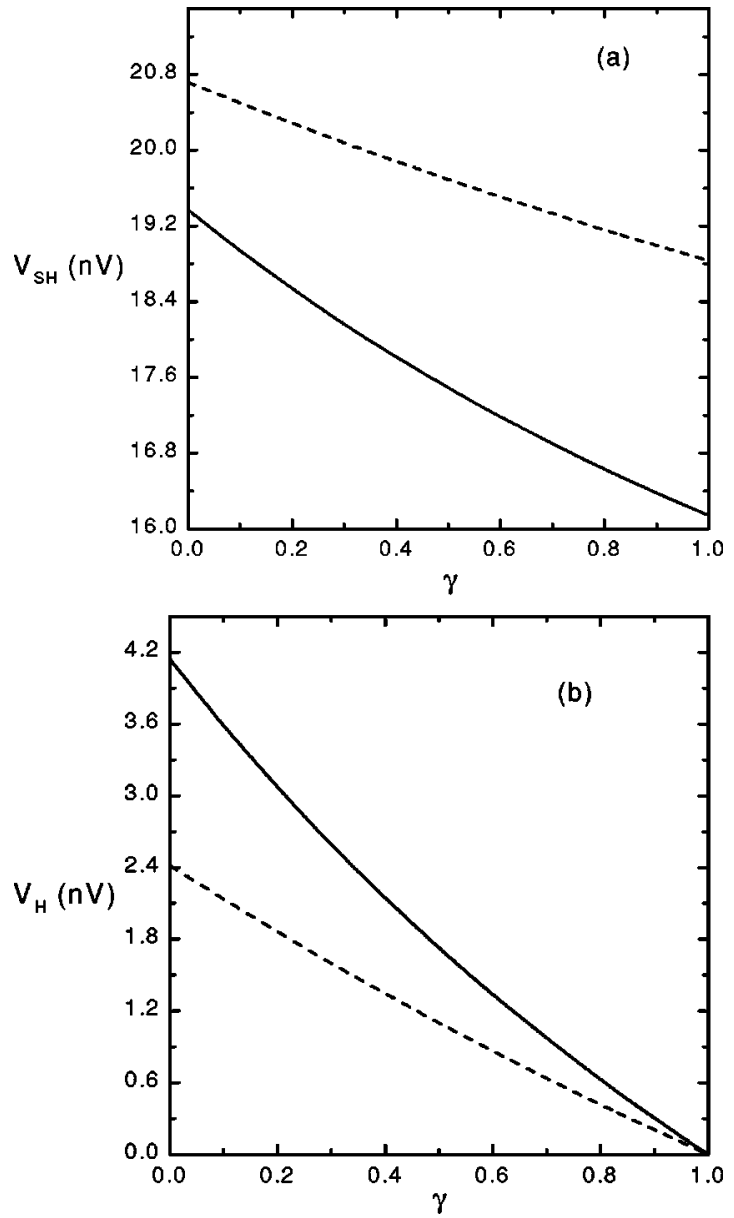

FIG. 3. Illustration of the changes of (a) the spin Hall voltage $V_{S H}$ and (b) the ordinary Hall voltage $V_{H}$ with the variation of the ratio $\gamma$ (i.e., the ratio of the "spin-flip" contact conductivity $\Sigma_{c}^{\prime}$ to the "spin-conserving" contact conductivity $\left.\Sigma_{c}\right) . \Sigma_{c}=0.2 C(C$ $=1 / \rho$ is the ordinary conductivity of $\mathrm{Al}$ ) for the solid line and $\Sigma_{c}$ $=0.1 \mathrm{C}$ for the dashed line. Other parameters are the same as used in Fig. 2.

$\Sigma_{c}^{\prime}$ to the spin-conserving contact conductivity $\Sigma_{c}$ ). From Figs. 3(a) and 3(b) one can see that the influences of spin-flip interfacial scattering on the spin Hall voltage $V_{S H}$ are not substantial, but spin-flip interfacial scattering may decrease significantly the ordinary Hall voltage $V_{H}$. From Fig. 3(b) one can see that $V_{H}$ will become very small if the spin-flip contact conductivity $\Sigma_{c}^{\prime}$ is comparable to the spin-conserving contact conductivity $\Sigma_{c}$. This can be seen directly from Eq. (26), from which one can see that $V_{H} \rightarrow 0$ in the limiting case of $\gamma \rightarrow 1$. In such cases, $V_{H}$ will be very small and may not be large enough to actually be measurable.

The second interesting case is that the width $L$ of the sample is much larger than the spin-diffusion length (i.e., $L$ $\gg D$ ). Below we will show that the spin Hall effect may be more easily detected in such cases. From Eqs. (23) and (24), one can show that if $L \gg D$, the ordinary Hall voltage between both edges of the transverse metal strip and the spin Hall voltage between both edges of the longitudinal sample will become 


$$
\begin{gathered}
V_{H}=\frac{2 \rho_{H} \rho_{H}^{\prime} \rho_{c} j_{x} l^{2} D(1-\gamma) e^{-L / 2 D} \cosh (y / D)}{\rho_{c}^{2} l^{2}+D l \rho_{c}(1+\gamma)\left(\rho+\rho^{\prime}\right)+4 \gamma D^{2} \rho \rho^{\prime}}, \\
V_{S H}=\frac{2 \rho_{H} j_{x} l D\left[\rho_{c}^{2} l+D \rho^{\prime} \rho_{c}(1+\gamma)\right]}{\rho_{c}^{2} l^{2}+D l \rho_{c}(1+\gamma)\left(\rho+\rho^{\prime}\right)+4 \gamma D^{2} \rho \rho^{\prime}} .
\end{gathered}
$$

From Eq. (31), one can see that in the case of $L \gg D$, the spin Hall voltage between both edges of the longitudinal sample will be independent of the width $L$ of the longitudinal sample, indicating that the spin Hall effect can be measured in macroscopic samples. This is the first merit of measuring the spin Hall effect in samples with $L \gg D$. Of course, in this case the ordinary Hall voltage $V_{H}$ between both edges of the transverse metal strip will depend on the width $L$ of the sample. However, if one measures $V_{H}$ at places near the ends of the transverse metal strip (i.e., at $y \simeq \pm L / 2$ ), the result will be

$$
V_{H} \simeq \frac{\rho_{H} \rho_{H}^{\prime} \rho_{c} j_{x} l^{2} D(1-\gamma)}{\rho_{c}^{2} l^{2}+D l \rho_{c}(1+\gamma)\left(\rho+\rho^{\prime}\right)+4 \gamma D^{2} \rho \rho^{\prime}} .
$$

This result is also independent of the width $L$ of the sample. Another merit to measuring the spin Hall effect in samples with $L \gg D$ is that the magnitudes of the ordinary Hall voltage $V_{H}$ and the spin Hall voltage $V_{S H}$ generated in samples with $L \gg D$ may be much larger than the corresponding values generated in samples with $L \ll D$, and hence can be more easily detected experimentally. To illustrate this, in Figs. 4(a) and 4(b) we have plotted the changes of $V_{S H}$ and $V_{H}$ (at $y$ $\simeq \pm L / 2$ ) systematically with the variation of the width $L$ of the longitudinal sample. From Figs. 4(a) and 4(b), one can see that $V_{S H}$ and $V_{H}$ both increase significantly as the width $L$ increases, and when $L$ is several times larger than the spindiffusion length $D, V_{S H}$ and $V_{H}$ will turn out to be constants (independent of the width $L$ ) and will be several times larger than the corresponding values obtained in the case of $L$ $\ll D$. These results suggest that the spin Hall effect may be more easily detected in the case of $L \gg D$ than in the case of $L \ll D$. Finally, one can show that in the case of $L \gg D$, the influences of contact resistances are similar, as is the case of $L \ll D$, illustrated in Figs. 2 and 3 . This can be see easily by making a detailed comparison between Eqs. (30) and (31) and (26) and (27).

In conclusion, we have discussed in detail the influences of spin transfer and contact resistances on the measurement of the spin Hall effect. We have shown that, due to the spin transfer between the longitudinal sample and the transverse metal strip, both the magnitudes of the spin Hall voltage produced in the longitudinal sample and the ordinary Hall
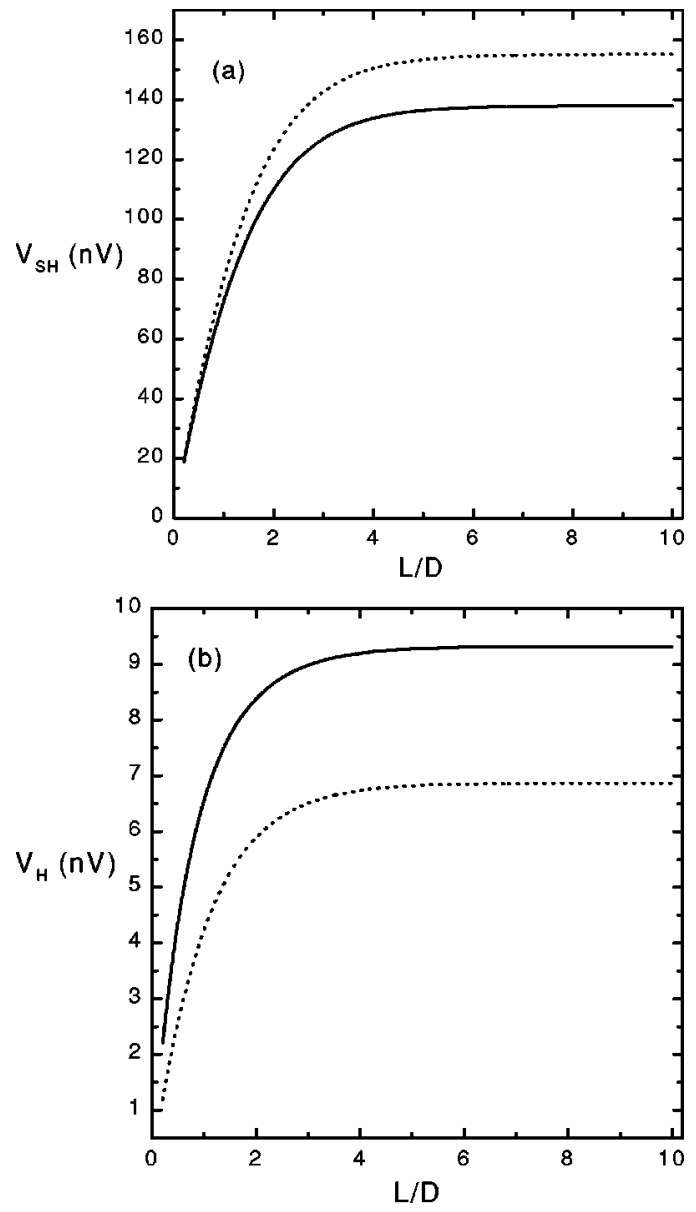

FIG. 4. Illustration of the changes of (a) the spin Hall voltage $V_{S H}$ and (b) the ordinary Hall voltage $V_{H}$ with the variation of the width $L$ of the longitudinal sample. $\rho_{c}=5 \rho$ ( $\rho$ is the ordinary resistivity of Al) for the solid line and $\rho_{c}=10 \rho$ for the dotted line. Other parameters are the same as used in Fig. 2.

voltage produced in the transverse metal strip, and the relation between them, will strongly depend on the contact resistance. The results show that in order to detect the spin Hall voltage correctly through the measurement of the ordinary Hall voltage, a clear understanding of the influences of spin transfer and contact resistance will be very important. It was also shown that it may be more appropriate to measure the spin Hall effect in samples with $L \gg D$ rather than in samples with $L \ll D$.

\section{ACKNOWLEDGMENTS}

This work was supported by a grant from the Research Grant Council of Hong Kong, China.
${ }^{1}$ J.E. Hirsch, Phys. Rev. Lett. 83, 1834 (1999).

${ }^{2}$ S. Zhang, Phys. Rev. Lett. 85, 393 (2000).

${ }^{3}$ M. Johnson and R.H. Silsbee, Phys. Rev. Lett. 55, 1790 (1985);

M. Johnson, ibid. 70, 2142 (1993).

${ }^{4}$ T. Valet and A. Fert, Phys. Rev. B 48, 7099 (1993).

\footnotetext{
${ }^{5}$ W.P. Pratt, Jr., S.F. Lee, J.M. Slaughter, R. Loloee, P.A. Schroeder, and J. Bass, Phys. Rev. Lett. 66, 3060 (1991).

${ }^{6}$ M.A.M. Gijs, S.K.J. Lenczowski, and J.B. Giesbers, Phys. Rev. Lett. 70, 3343 (1993).

${ }^{7}$ C.L. Chien and C.R. Westgate, The Hall Effects and Its Applica-
} 
tions (Plenum, New York, 1980), Chaps. 1-4, and references therein.

${ }^{8}$ C.M. Hurd, The Hall Effects in Metals and Alloys (Plenum, New York, 1973), Chap. 5, and references therein.

${ }^{9}$ G.A. Prinz, Phys. Today 48 (4), 58 (1995); S.A. Wolf et al., Science 294, 1488 (2001).

${ }^{10}$ It is worth noting that the spin current discussed here carries no charge, unlike the spin-polarized charge current discussed extensively in literatures. See S.Q. Shen and X.C. Xie, Phys. Rev. B
67, 144423 (2003).

${ }^{11}$ E.I. Rashba, Phys. Rev. B 62, R16267 (2000); Eur. Phys. J. B 29, 513 (2002).

${ }^{12}$ From Eqs. (1) and (2), the ordinary resistivity $\rho$ and $\rho^{\prime}$ and the anomalous Hall resistivity $\rho_{H}$ and $\rho_{H}^{\prime}$ should be given by $\rho$ $=C /\left(C^{2}+C_{H}^{2}\right), \rho_{H}=C_{H} /\left(C^{2}+C_{H}^{2}\right), \rho^{\prime}=C^{\prime} /\left(C^{\prime 2}+C_{H}^{\prime 2}\right)$, and $\rho_{H}^{\prime}=C_{H}^{\prime} /\left(C^{\prime 2}+C_{H}^{\prime 2}\right)$. Since $C_{H} \ll C$ and $C_{H}^{\prime} \ll C^{\prime}$, one has $\rho$ $=1 / C, \rho_{H}=C_{H} / C^{2}, \rho^{\prime}=1 / C^{\prime}$, and $\rho_{H}^{\prime}=C_{H}^{\prime} / C^{\prime 2}$. 